\title{
Personal attribute
}

\author{
Robert West ${ }^{1}$ \\ 1 University College London, University of London
}

A specifically dependent continuant that inheres in a human being.

Curator note: 'Specifically dependent continuant' is a high level entity in basic Formal Ontology (BFO) https://basic-formal-ontology.org/

Elaboration: Includes the BFO entities qualities, dispositions and roles. It includes anything that might characterise an person. 